$\xi=-1$

\title{
Solving Dual Fuzzy Nonlinear Equations via Shamanskii Method
}

\author{
Ibrahim Mohammed Sulaiman ${ }^{1}$, Mustafa Mamat $^{1 *}$, Nurnadiah Zamri $^{1}$, Puspa Liza Ghazali ${ }^{2}$ \\ ${ }^{1}$ Faculty of Informatics and Computing, Universiti Sultan Zainal Abidin, Terengganu, Malaysia \\ ${ }^{2}$ Faculty of Economy and Management Sciences, Universiti Sultan Zainal Abidin, Terengganu, Malaysia \\ *Corresponding author E-mail: must@ unisza.edu.my
}

\begin{abstract}
New ideas on numerical methods for solving fuzzy nonlinear equations have spread quickly across the globe. However, most of the methods available are based on Newton's approach whose performance is impaired by either discontinuity or singularity of the Jacobian at the solution point. Also, the study of dual fuzzy nonlinear equations is yet to be explored by many researchers. Thus, in this paper, a numerical method to investigate the solution of dual fuzzy nonlinear equations is proposed. This method reduces the computational cost of Jacobian evaluation at every iteration. The fuzzy coefficients are presented in its parametric form. Numerical results obtained have shown that the proposed method is efficient.
\end{abstract}

Keywords: Fuzzy nonlinear equations; numerical methods; parametric form; Shamanskii method.

\section{Introduction}

Nonlinear equations are undoubtedly one of the domains in which fuzzy set theorem assume a central position. The development of methods for numerical evaluations was a response to the demand of numerical computation, mainly in systems nonlinear equations. However, most of the parameters from these systems are usually represented by fuzzy numbers rather than crisp numbers. Thus, the outcome depends on the roots of fuzzy equations [2]. Some standard analytical techniques such as [3, 4] are proposed for solving fuzzy equations. Numerical investigations have shown that these methods are only able to solve linear and algebraic fuzzy equations, but not suitable for solving fuzzy nonlinear equations such as:

$$
\begin{aligned}
& \text { (1) } a x^{4}+b x^{3}+c x^{2}+d x+e=f \\
& \text { (2) } a e^{x}+b=d
\end{aligned}
$$

where $x, a, b, c, d, e$ and $f$ are fuzzy numbers. Therefore, there is need to develop numerical methods for solving fuzzy nonlinear equations. New ideas spread quickly which lead to publications on numerical methods for the solution of fuzzy nonlinear equations. In [1] parameterized some fuzzy quantities and applied Newton's approach to solve the equivalent fuzzy nonlinear equations. The Newton's method was further extended to solve dual fuzzy nonlinear equations by [15]. In [14] suggested a Jacobian updating formula for the Shamanskii method and applied it to solve fuzzy nonlinear equations having singular Jacobian. In [16] employ the Chord's Newton method to solve dual fuzzy nonlinear equations. In [13] further solved dual fuzzy nonlinear problems by Levenberg-Marquardt approach. The solution of fuzzy nonlinear equation was investigated using Broyden's method by [2]. Broyden and Chord Newton methods are variant of Newton's method with better convergence properties [5]. However, the performance of these methods are affected by Jacobian or approximate Jacobian computation in all iterations. Also, the performance may be impaired by the singularity of the Jacobian at the solution points $[8,9]$. Recently, in $[11,12]$, applied the Regula Falsi method to solve fuzzy nonlinear equation. In a major revolution in numerical practice, we suggested the Shamanskii's method for the solution of dual fuzzy nonlinear equation. This method starts by computing the Newton step in the first iteration and followed by several Chord steps. This will reduce the computational burden of the Jacobian matrix at every iteration, unlike Newton's type methods. Moreover, if the initial guess is close to the solution point and the Jacobian been nonsingular, then this proposed method will converge q-superlinearly [9]. The applications of fuzzy systems can be refer to $[18,19]$.

This paper is structured as follows. Section 2 discusses the overview and some fundamental of fuzzy numbers. In section 3 , we present the Shamanskii method for solving dual fuzzy nonlinear equations. Numerical illustration on well-known problem is presented in section 4. Finally, we present the conclusion in section 5.

\section{Preliminaries}

In this section, we recall useful definitions of fuzzy numbers.

Definition 2.1. A fuzzy number is a set like $u: R \rightarrow I=[0,1]$, which satisfy the following $[6,17]$ :

1. $u$ is upper semi-continuous

2. $u(x)=0$ outside some interval $\left[c_{v} d\right]$

3. There are real numbers $a_{x} b$ such that $c \leq a \leq b \leq d$ and

$3.1 u(x)$ is monotonic increasing on $\left[c_{x} a\right]$

$3.2 u(x)$ is monotonic deceasing on $\left[b_{v} d\right]$

$3.3 u(x)=1_{x} a \leq x \leq b$ 
The set of all fuzzy numbers is denoted by $E$. An equivalent parametric is also given in [7].

Definition 2.2. Fuzzy number $u$ in parametric form is a pair $(\underline{u}, \bar{u})$ of function $\underline{u}(\alpha), \bar{u}(\alpha), 0 \leq \alpha \leq 1$, which satisfies the following requirement:

(1) $\underline{u}(\alpha)$ is a bounded monotonic increasing left continuous function,

(2) $\bar{u}(\alpha)$ is a bounded monotonic decreasing left continuous function,

(3) $\underline{u}(\alpha), \bar{u}(\alpha), 0 \leq \alpha \leq 1$

Definition 2.3. A popular fuzzy number is the triangular fuzzy number $u=(a, b, c)$ with membership function [6, 17].

$u(x)= \begin{cases}\frac{(x-a)}{(c-a)}, & a \leq x \leq c_{x} \\ \frac{(x-b)}{(c-b)}, & c \leq x \leq b_{x}\end{cases}$

where $c \neq a, c \neq b$. Its parametric form is

$\underline{u}(\alpha)=a+(c-a) \alpha, \quad \bar{u}(\alpha)=b+(c-b) \alpha$

Let $T F(\mathbb{R})$ be the set of all trapezoidal fuzzy numbers. The addition and scalar multiplication of fuzzy numbers are defined by the extension principle and can be equivalently represented as follows [1].

For arbitrary $u=\left(\underline{u}_{v}, \bar{u}\right), v=\left(\underline{v}_{v}, \bar{v}\right)$, and $k>0$, the addition $(u+v)$ and multiplication by scalar $k$ are defined as

$(\underline{u+v})(\alpha)=\underline{u}(\alpha)+\underline{v}(\alpha)_{0} \cdot(\overline{u+v})(\alpha)=\bar{u}(\alpha)+\bar{v}(\alpha)_{x}$ $(\underline{k u})(\alpha)=k \underline{u}(\alpha),(\overline{k u})(\alpha)=k \bar{u}(\alpha)$

\section{Shamanskii method for solving dual fuzzy nonlinear equations}

Shamanskii method [10] lies between Newton's method and Chord method. This method move through an intermediate sequence says $\left\{w_{k p}\right\}_{p=1}^{m}$, which is one Newton's step followed by several Chord step [8]. The Shamanskii method is defined as follows.

Consider a dual fuzzy nonlinear equation

$F(x)=G(x)+c$

The parametric form is given as

$$
\left\{\begin{array}{l}
\underline{F}\left(\underline{x}_{v} \bar{x}_{v} r\right)=\underline{G}\left(\underline{x}_{v} \bar{x}_{v} r\right)+\underline{c}(r), \\
\bar{F}\left(\underline{x}_{v} \bar{x}_{v} r\right)=\bar{G}\left(\underline{x}_{s} \bar{x}_{v} r\right)+\bar{c}(r) .
\end{array} \quad \forall r \in[0,1]\right.
$$

To obtain the solution of the above equation by Shamanskii's method, we start with a given initial point $x_{0}$ and generate a sequence of points $\left\{x_{k}\right\}$ that converge to the solution $x^{*}$. This method can be described by the following algorithm.

\footnotetext{
Algorithm 1: Shamanskii Method for Dual Fuzzy Nonlinear Equation (SMFDNE)

Step 1: Given a dual fuzzy nonlinear equation, transform it into parametric form

Step 2: Compute $F\left(x_{k}\right)$

Step 3: Compute $W_{k i 1}=x_{k}-J^{-1} F\left(x_{k}\right)$

Step 4: Set $J\left(x_{k}\right) \& J\left(x_{0}\right) \forall \mathbb{k}$
}

Step 5: Update $W_{k p+1}=x_{k, p}-J^{-1} F\left(W_{k p p}\right)$,

$$
\begin{aligned}
& k=1,2_{v a x} \text { and } p \in(1, m-1) \\
& x_{k+1}=W_{k, m}
\end{aligned}
$$

Step 6: Repeat Steps 3 to 5 and continue with the next $k$ until tolerance criteria $\epsilon \leq 10^{-4}$ are satisfied.

For $m=1$, we have Newton's method, and $m=\infty$, we obtain the chord method [9].

\section{Results and discussion}

In this section, we present the solution of a dual fuzzy nonlinear problem using the proposed Shamanskii method to illustrate its efficiency. Also, the solution is plotted in Figure 1 and Figure 2 respectively.

Example 1. Consider the following fuzzy nonlinear problem.

$$
(4,10,16) x^{2}+(0,1,2) x=+(2,4,6) x^{2}+(1,2,3)
$$

Without loss of generality, let $X$ be positive and hence the parametric form of the above fuzzy nonlinear equation is defined as:

$$
\begin{aligned}
& (4+6 r) x^{2}(r)+r \underline{x}(r)=(2+2 r) \underline{x}^{2}(r)+(1+r) \\
& (16-6 r) \bar{x}^{2}(r)+(2-r) \bar{x}(r)=(6-2 r) \bar{x}^{2}(r)+(3-r)
\end{aligned}
$$

which can be rewritten as

$$
\begin{aligned}
& (2+4 r) x^{2}(r)+r x(r)-(1+r)=0, \\
& (10-4 r) \bar{x}^{2}(r)+(2-r) \bar{x}(r)-(3-r)=0
\end{aligned}
$$

Next, we compute the Jacobian as follows.

$$
I\left(\underline{x}_{v} \bar{x}_{v} r\right)=\left[\begin{array}{cc}
2(2+4 r) \underline{x}(r)+r & 0 \\
0 & 2(10-4 r) \bar{x}(r)+(2-r)
\end{array}\right]
$$

To obtain the initial guess, we solve the above equations for $r=0$ and $r=1$. For $r=0$, we have

$2 x^{2}(0)-1=0$,

$1 \overline{0} \bar{x}^{2}+2 \bar{x}(0)-3=0$.

And for $r=1$, we get

$6 \underline{x}^{2}(1)+\underline{x}(1)-2=0$

$6 \overline{\bar{x}}^{2}(1)+\overline{\bar{x}}(1)-2=0$.

After the computations, we got $\underline{x}(0)=0.7071_{i} \bar{x}(0)=0.4567$, and $\underline{x}(1)=0.5000$ and $\bar{x}(1)=0.5000$. From definition of the initial guess,

$x_{0}=(\underline{x}(0) s \underline{x}(1) s \bar{x}(0))$,

Then, we have

$x_{0}=\left(0.7071_{v} 0.5000,0.4567\right)$.

However, this initial guess is close to the exact solution. Thus, to illustrate the efficiency of the proposed methods, we propose $x_{0}=(0,8,0,6,0.5)$ as the new initial guess. The solution was obtained after 2 iterations using maximum error less than $10^{-4}$ (refer Figure 1 and Figure 2 for details of the solution). 


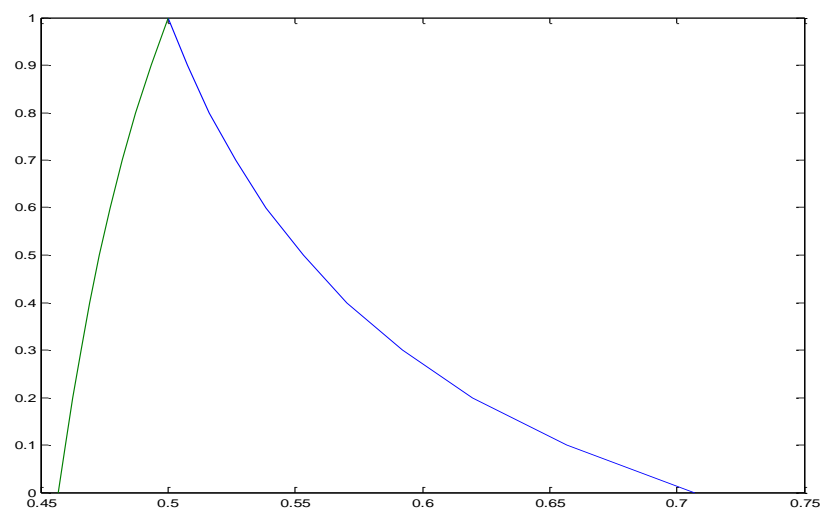

Fig. 1: Positive solution of Example 1.

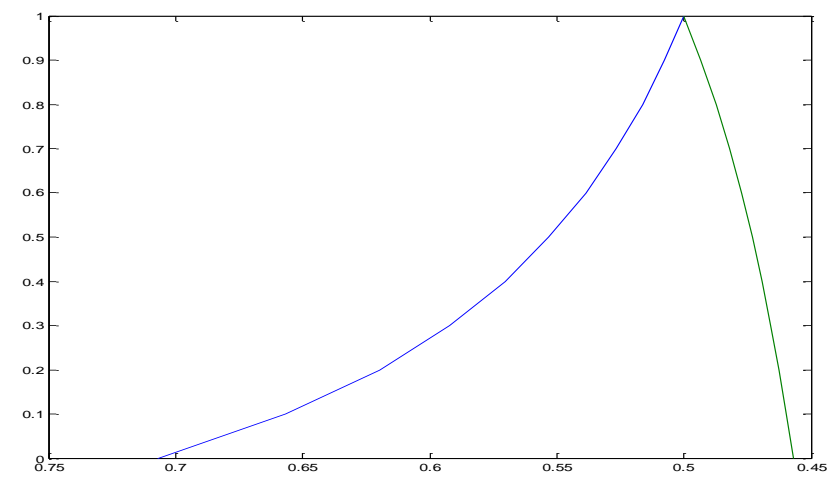

Fig. 2: Reversed Positive solution of Example 1.

\section{Conclusion}

Fuzziness often occurs in the mathematical modelling of realworld systems. This is due to the complexity of the problem being modelled. Numerous research and publications most of which have advanced this field in many areas including fuzzy numbers, fuzzy equations, and many more. However, the study of solution methods for fuzzy and dual singular fuzzy nonlinear equations has not been done by many researchers. In this paper, we present an efficient numerical algorithm for solving dual fuzzy nonlinear equations. This method computes the Jacobian matrix once throughout the iteration process. Its efficiency and less computations to solve dual fuzzy problems make it a good alternative.

\section{Acknowledgement}

The authors would like to thank the government of Malaysia for funding this research under the Fundamental Research Grant Scheme (FRGS/1/2017/STG06/Unisza/01/1) and also Universiti Sultan Zainal Abidin, Terengganu, Malaysia.

\section{References}

[1] Abbasbandy, S., Asady, B. "Newton Method for solving fuzzy nonlinear equations" Applied Mathematics and Computation, 159 (2004) $349-356$.

[2] Amirah, R., Lazim, M., Mamat, M. "Broyden's method for solving Fuzzy nonlinear equations" Advance in Fuzzy System, (2010), Article ID 763270, 1-6.

[3] Buckley J.J., Qu Y. "Solving fuzzy equations: A new solution concept" Fuzzy Set and Systems, 39 (1991) 291- 301.

[4] Buckley J.J., Qu Y. "Solving linear and quadratic fuzzy equations" Fuzzy Sets and Systems, 38 (1990) 43 - 59.

[5] Chong, E. K. P., Zak, S. H. An introduction to optimization. Wiley Series in Discrete Mathematics and Optimization, 2013.

[6] Dubois, D., Prade, H. Fuzzy Sets and Systems. Theory and Application. Academic Press, New York, 1980.

[7] Goetschel, R., Voxman, J.W. "Elementary fuzzy calculus," Fuzzy set and Systems, 18 (1986) 31-43.
[8] Kelley C. T. "Iterative Methods for Linear and Nonlinear Equations". SIAM, Philadelphia, 1995.

[9] Kelley C. T. "A Shamanskii-Like Acceleration Scheme for Nonlinear Equations at Singular Roots" Mathematics of Computation, 47 (1986), 609-623.

[10] Shamanskii, V.E. "A modification of Newton's method” Ukrain. Mat. Zh., 19 (1967), 133-138.

[11] Sulaiman, I.M. New Iterative Methods for Solving Fuzzy and Dual Fuzzy Nonlinear Equations. Universiti Sultan Zainal Abidin, Terengganu, Malaysia, PhD Thesis, 2018.

[12] Sulaiman, I. M., Mamat, M., Waziri, M. Y., Fadhilah, A., Kamfa, U. K. Regula Falsi Method for Solving Fuzzy Nonlinear Equation. Far East Journal of Math Sci., 100, 6, (2016), 873-884.

[13] Sulaiman, I. M, Mamat, M., Waziri, M. Y, Mohamed, M.A., Mohamed, F.S. Solving Fuzzy Nonlinear Equation via LevenbergMarquardt method. Far East Journal of Math Sci., 103, 10, (2018), 1547-1558.

[14] Sulaiman, I. M, Mamat, M., Mohamed, M.A., Waziri, M. Y. Diagonal Updating Shamasnkii-Like method for Solving Fuzzy Nonlinear Equation. Far East Journal of Math Sci., 103, 10, (2018), 1619. 1629.

[15] Tavassoli, M. K., Abbasbandy, S., and Hadi, A. V. An Iterative Method for solving Dual fuzzy nonlinear equations. Applied Mathematics and Computation, 167 (2005), 316-323.

[16] Waziri M.Y., Moyi A. "An alternative approach for solving dual fuzzy nonlinear equations International Journal of Fuzzy Systems, 18 (2016), $103-107$.

[17] Zimmermann H. J. Fuzzy Set Theory and its Applications. Third ed., Kluwer Academic, Norwell, MA 1991.

[18] Mamat, M., Deraman, S.K., Noor, N.M.M., Rokhyati, Y. Diet problem and nutrient requirement using fuzzy linear programming approach, Asian Journal of Applied Sciences, 5(1): 52-59

[19] Mamat, M., Rokhayati, Y., Noor, N.M.M., Mohd, I. Optimizing human diet problem with fuzzy linear programming approach (2011), Pakistan Journal of Nutrition, 10(6): 594-598. 